\title{
Aggressive periodontitis
}

\author{
Siebert T, Malachovsky I, Mikuskova K, Janickova M \\ Department of Stomatology and Maxillofacial Surgery, Jessenius Faculty of Medicine in Martin, Comenius \\ University in Bratislava, University Hospital in Martin, Slovakia. maria.janickova @ uniba.sk
}

\begin{abstract}
AIM: Horizontally impacted mandibular molars may cause loss of bone, and development of periodontal pockets on the distal root surface of adjacent second molars. The reported patient was confirmed to have aggressive periodontitis. The aim of this presentation is to describe a novel view of a complex treatment approach to promote periodontal healing in a patient.

MATERIAL AND METHODS: Our study presents the results of a patient with generalized aggressive periodontitis, horizontally impacted left third mandibular molar, and a second molar with a deep periodontal pocket. The treatment concept was recommended based on the idea of "one-stage treatment". The removal of the third molar was followed by deep scaling and root planing, and the xenogenic grafting material was placed on the bone defect. The flap completely covered the wound. The patient received systemic antibiotics. RESULTS: The probing pocket depth was $9 \mathrm{~mm}$ before surgical treatment and 0-2 mm 1, 5, and 10 years postoperatively. The radiographic bone level was $50 \%$ before surgery and $100 \%$ after the surgical approach. CONCLUSION: This presentation with a 10-year follow-up describes the implementation of one-stage treatment management to promote periodontal healing in a patient via full-mouth periodontal and surgical therapy (Fig. 4, Ref. 33). Text in PDF www.elis.sk

KEY WORDS: impacted third molar, aggressive periodontitis, guided tissue regeneration, one-stage treatment.
\end{abstract}

\section{Introduction}

Periodontal health should be defined as a state free from inflammatory periodontal disease that allows an individual to function normally and not suffer any consequences (mental or physical) as a result of past disease (1). Periodontal health is represented by the stability, aesthetics and function of the teeth as a source of social quality of life.

Periodontitis is a chronic multifactorial inflammatory disease that is associated with dysbiotic plaque biofilms and is characterized by progressive destruction of the tooth-supporting apparatus (2). Its primary features include the loss of periodontal tissue support manifested by clinical attachment loss (CAL), radiographically assessed alveolar bone loss, presence of periodontal pocketing and gingival bleeding (2). Generalized aggressive periodontitis (GAgP) is characterized as "generalized interproximal attachment loss affecting at least three permanent teeth other than the first molars and incisors" (3).

Periodontitis is a major public health problem due to its high prevalence, as well as because it may lead to tooth loss and later

Department of Stomatology and Maxillofacial Surgery, Jessenius Faculty of Medicine in Martin, Comenius University in Bratislava, University Hospital in Martin, Slovakia

Address for correspondence: $\mathrm{M}$. Janickova, MD, $\mathrm{PhD}$, MPH, University of Komensky, Jessenius Faculty of Medicine, Department of Stomatology and Maxillofacial Surgery, Jessenius Faculty of Medicine in Martin, Comenius University in Bratislava, University Hospital in Martin, Slovakia, Kollarova 2, SK-036 01 Martin, Slovakia

Phone No.: +421.43 .4203517$ to the loss of all dentitions. This can lead to significant dental care costs and negative impacts on general health.

Many complications can occur during the eruption of the lower mandibular third molar (3M), such as maintaining its stable position with a poor growth direction and remaining impaction in the lower jaw. The anatomy, position, and relationships of teeth within the dental arches are among the factors that have been associated with plaque retention, gingivitis, and periodontitis (4). Other complications may occur during surgical extraction of the lower mandibular third molar in conventional surgical removal methods.

Furthermore, extraction may cause loss of bone, development of periodontal pockets, and exposure of the cementum on the distal root surface of the adjacent second molar (2M), all of which may unfavourably affect the long-term prognosis of the tooth (5)

Thus, these conventional surgical procedures may cause recession of the gingiva and hyperesthesia of dentin with spontaneous pain.

This narrative study summarizes the current evidence about the role of augmentation procedures and their benefits to a patient with GAgP and impacted 3M.

\section{Clinical study}

For this study, we aimed to use the novel approach of guided tissue regeneration in a patient with aggressive periodontitis and a horizontally impacted mandibular $3 \mathrm{M}$.

Although the impacted mandibular $3 \mathrm{M}$ is a purely oral surgical problem, we performed an overall complex examination of the patient's oral cavity. 


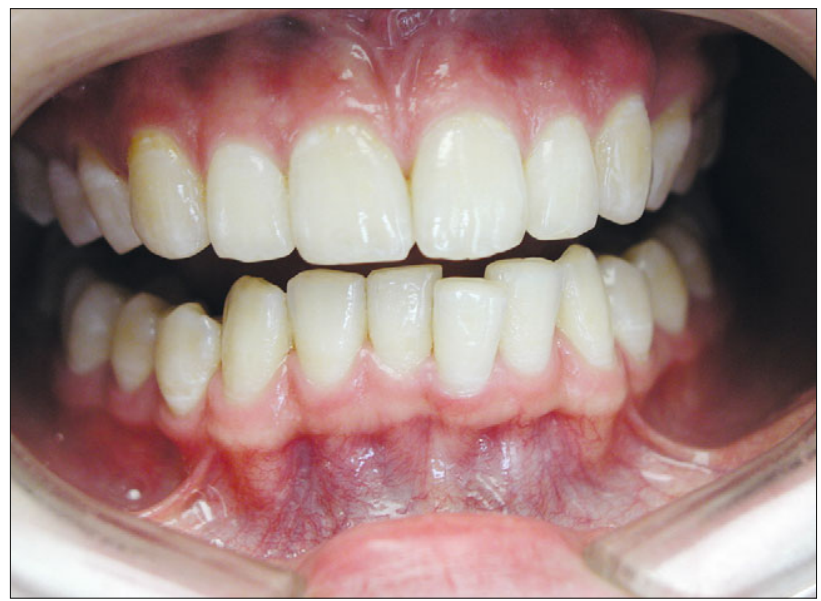

Fig. 1. A 26-year-old patient with GAgP.

This study focuses not only on the destruction of periodontium of adjacent $2 \mathrm{M}$, but also on GAgP. The most recent effort to classify AgP was presented in 1999 in form of a report by the committee on the classification of periodontal diseases of the American Academy of Periodontology (AAP) (6). Tooth-related anatomical factors such as root abnormalities and fractures, and tooth relationships within the dental arch as well as with the opposing dentition can enhance plaque retention (7).

The overall oral hygiene status of the patient with aggressive periodontitis is good, as assessed by PLI (oral hygiene index; O'Leary 1972). The periodontal health of the patient was assessed as maximum PLI of $15 \%$. By GAgP, we can monitor the minimal amount of calculus and plaque (Fig. 1). Monitoring health or inflammation of the gingival tissues is best documented by the parameter of BoP. Subsequent studies investigated the predictive value of absence of $\mathrm{BoP}$ as an indicator for periodontal stability (1).

The previous clinical and histological data suggest that bleeding is an initial sign of GAgP, which precedes the visual signs of inflammation like redness and swelling. PPD or probing attachment levels alone should not be used as evidence of gingival health or disease. They must be considered in conjunction with other important clinical parameters such as BoP, as well as with the modifying and predisposing factors (1)

In our study, the initial examination of a 26-year-old patient revealed a pocket with a probing pocket depth (PPD) of 6-9 mm, presence of gingival bleeding on probing (BoP index; Ainamo 1975 ) of $86 \%$, and PLI of $14 \%$. The pockets were particularly deep in the molar and incisor regions, while the premolar region was slightly less involved. The PPD at the distal location reached $9 \mathrm{~mm}$ while at the buccal and lingual locations of the distal root surface of the second molar (tooth \#37), it measured $8 \mathrm{~mm}$.

Altogether, there were minimal signs of inflammation other than BOP. Familial aggregation of GAgP was ruled out.

The panoramic radiograph (Fig. 2) showed the presence of a fully horizontally impacted left mandibular 3M (tooth \#38), with the mesial cusp lying laterally and close to $50 \%$ of the distal root of the second molar (tooth \#37). We observed well-defined radiolucency surrounding the crown of the impacted tooth and involving the distal root of the neighbouring $2 \mathrm{M}$.

Crestal bone loss at the distal surface of $2 \mathrm{M}$ was also measured on panoramic radiograph, with the distance from the cementoenamel junction to the bottom of the defect being $8 \mathrm{~mm}$. Localized vertical defects that extended up to $15 \%$ of the root length could be observed on several teeth of the lower jaw.

Based on the history, examination findings, and radiographic findings, the diagnosis of GAgP was made according to the criteria of the Armitage classification (2004).

The patient was informed about the aetiology of periodontal disease and instructed on plaque control methods. During the initial treatment phase, supragingival plaque was removed, and the teeth were polished. Microbiological analysis of the subgingival plaque samples collected from the deepest sites revealed elevated levels of Aggregatibacter actinomycetemcomitans (A.a.) as well as Porphyromonas gingivalis (P.g.).

Due to the microbiological analysis, we continued after two weeks with mechanical debridement and surgical treatment in form of ana one-stage treatment approach. Deep scaling and root planning were performed under local anaesthesia in the mandible. At the same time, under local anaesthesia, we prepared a full-thickness mucoperiosteal flap at a distance of $2 \mathrm{M}$ distally to the retromolar region, with no vertical releasing incisions. After flap exposure, we divided the left third mandibular molar into several parts without destroying the buccal and lingual alveolar bone proper. The remaining extraction socket was a 1-walled defect rather than a traditional 4-walled socket defect, as the buccal and lingual bone walls were completely absent. At the time of surgical exposure of $3 \mathrm{M}$, root surface debridement was performed at the distal aspect of the adjacent $2 \mathrm{M}$. We placed xenogenic $\beta$-tricalcium phosphate augmentation material (CERASORB M, Curasan, Germany) as a guided tissue regeneration (GTR) method. The one-stage treatment approach was followed by a systemic antibiotic regimen (amoxicillin + clavulanic acid, $1000 \mathrm{mg}$ twice daily, and metronidazole, $1000 \mathrm{mg}$ daily, for one week) for a surgical approach with treatment of GAgP, which we started 1 day before the treatment.

Healing was uneventful, and postoperative clinical and radiographic examinations after the extraction revealed good clinical

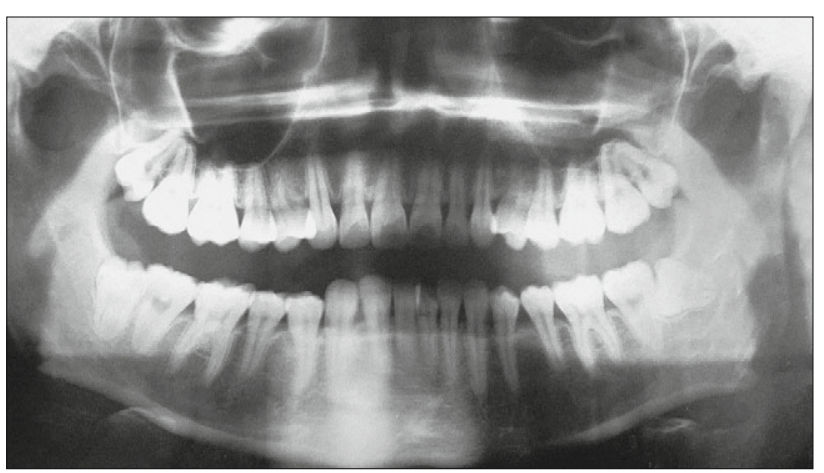

Fig. 2. Initial panoramic radiograph. Note the destruction of the distal aspect of tooth \#37. 


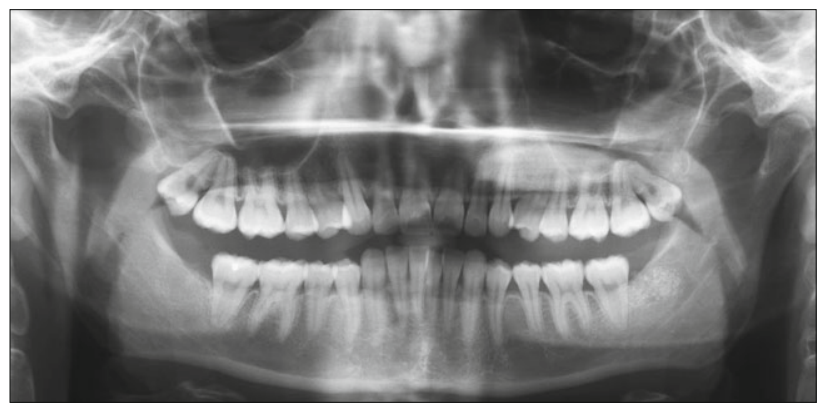

Fig. 3. Panoramic radiograph, 1 year after one-stage treatment.

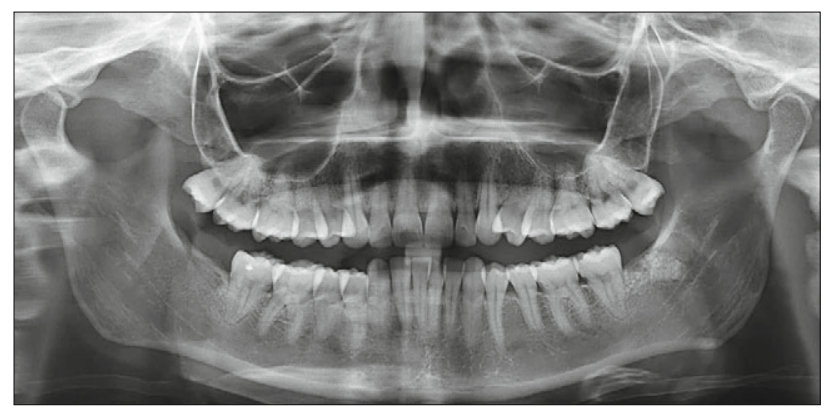

Fig. 4. Panoramic radiograph, 10-year follow up.

results with no residual bone defect at the distal surface of $2 \mathrm{M}$. At 1-year, 5-year and 10-year re-evaluations, the 2M PPD values were $2 \mathrm{~mm}$ at the disto-lingual location and $2 \mathrm{~mm}$ at the distobuccal location. The oral hygiene status of the patient was still good according to oral hygiene index (PLI $=\%$ ) and gingival index $(\mathrm{BoP}=7.14 \%)$. No crestal bone loss was observed on the distal surface of $2 \mathrm{M}$.

Radiographs obtained 1 (Fig. 3), 5 and 10 years after therapy (Fig. 4) showed filling of the bony defects and sharp contours of the gained hard tissues.

The patient was enrolled in a supportive periodontal therapy programme.

\section{Discussion}

The symptoms of aggressive periodontitis ( $\mathrm{AgP})$ are specific, making the correct diagnosis difficult and dependent on clinical manifestations and radiographic scans.

AgP comprises a group of rare, often severe, rapidly progressive forms of periodontitis, often characterized by clinical manifestation at an early age and a distinctive tendency for cases to aggregate in families (3) GAgP is a rapidly progressive disease that affects otherwise healthy individuals, and results in a rapid loss of attachment and bone destruction, which may lead to edentulism early in life (11). In this study, we investigated a general healthy 26-year-old patient, and at that time, no dental professionals knew about the patient's GAgP. We have to protect young patients because the loss of teeth and whole dentition can lead to a disruption of organism balance and has important social and economic implications. The treatment of this condition has always represented a challenge for clinicians because there are no established protocols and guidelines for efficiently controlling the disease (12).

Our treatment plans have been centred on a one-stage treatment approach, which means mechanical deep scaling and root planing on the same day combined with a surgical protocol for $3 \mathrm{M}$, systemic antibiotics for both procedures and effective supragingival plaque control in supportive periodontal treatment. We started the treatment of GAgP with scaling and root planing in combination with systemic antibiotics; the initiation of antibiotic therapy was performed $24 \mathrm{~h}$ before scaling and root planning. The surgical approach was implemented simultaneously with the treatment, during which the antibiotic was prescribed. The presence of periodontal pathogens, specifically A.a. and P.g, has been implicated as the reason why aggressive periodontitis does not respond to conventional therapy without antibiotics. One-stage full-mouth disinfection (OSFMD) has been proposed as a therapeutic approach that is particularly indicated for patients who experience advanced and rapidly progressing periodontal diseases $(13,14,15)$. Adjunctive antimicrobial therapy with systemic antibiotics kills bacteria outside the range of root surface instrumentation and affects periodontal pathogens residing in nonperiodontal mucosal surfaces (16). Recent reports from the European Federation of Periodontology, and American Academy of Periodontology suggest that patients with GAgP appear to benefit from adjunctive use of systemic antibiotics $(17,18)$.

Periodontal infections contain a wide variety of bacteria; hence, no single antibiotic can be effective against all putative pathogens (19). Among the possible regimens, the combination of amoxicillin and metronidazole may be a more effective therapy because of the synergistic effect of this combination and its wide spectrum of activity $(12,18,20)$.

To the best of our knowledge, a limited number of randomized controlled clinical trials have been focused on the additional effects of adjunctive amoxicillin-metronidazole in non-surgical treatment of GAgP patients, while no information is available regarding the added benefits for OSFMD $(11,19,20)$.

In the current study, we examined and observed a 10-year clinical effect and radiographic results of a special surgical approach with GTR procedures after $3 \mathrm{M}$ removal and use of antibiotics indicated for the treatment of GAgP.

This study investigated the periodontal pockets of the mandibular 2Ms, while making comparisons before conventional extraction of the enclosed adjacent mandibular 3M. Tooth-related anatomical factors, root abnormalities, and fractures can act as plaque-retentive factors and increase the likelihood of gingivitis and periodontitis (7). The presence of preoperative bone defects is a risk factor for persistent postoperative infrabony defects (20). We must always consider orthodontics with expansion therapy of the whole lower arch as an alternative to $3 \mathrm{M}$ extraction before the surgical treatment. The question of extraction versus expansion therapy has been a frequent topic of various discussions (21)

Some studies demonstrated that there was an improvement in probing depth on the distal surface of $2 \mathrm{Ms}$ after extraction of the adjacent $3 \mathrm{M}(22,23,24)$. However, a number of authors have previously reported that extraction of $3 \mathrm{Ms}$ can lead to alterations 
in the periodontal condition of $2 \mathrm{Ms}$, particularly on the distal surface $(25,26)$.

In our study, we planned to avoid the formation and enlargement of the periodontal pocket on $2 \mathrm{M}$ after surgery because we used a novel surgical approach.

This study also examined the periodontal condition of the adjacent mandibular $2 \mathrm{M}$ and compared the conditions at the initial clinical examination and 10 years after surgery. All previous studies of similar approaches assessed results after 1 year, with the maximum of 5 years $(20,22,23)$. This study is unique in that it yields results after 10 years of follow-up from the start of treatment.

It has previously been hypothesized that the type of flap used during the extraction of $3 \mathrm{M}$ affects the periodontal condition of the adjacent $2 \mathrm{M}$ after surgery. However, several studies have compared different types of flaps in relation to the extraction of $3 \mathrm{M}$ and concluded that the periodontal tissue of $2 \mathrm{M}$ is significantly different after the extraction of $3 \mathrm{M}(27,28,29)$.

Thus, it is believed that the surgical technique applied in the present study, a flap with no vertical incision, did affect the results obtained.

After 3M removal, we used a modified method of known GTR techniques. We needed all measures to prevent or reduce bone resorption after tooth extraction. This modified approach has been developed to minimize bone loss, especially in the zone after extraction of the third mandibular molar and the periodontally developed pocket on the adjacent second mandibular molar. The use of GTR therapy has been proposed to enhance periodontal healing after $3 \mathrm{M}$ removal $(30,31)$.

This method used xenogenic bone fillers as biomaterial. Ideally, the therapeutic plan starts before tooth extraction, and offers two options: spontaneous healing of the extraction socket, which can lead to bone loss of the alveolar bone and distal aspect with hypersensitivity of $2 \mathrm{M}$, or techniques for preserving the alveolar bone that develop new bone to fill the defect. Therefore, the volume is preserved, and the collapse of surrounding tissues is avoided.

The characteristics for ideal bone replacement graft materials include safety, efficacy, and ability of the graft material to be replaced by vital alveolar bone. The beta-TCP graft material used in this study is a purely synthetic material fabricated to exact chemical specifications (32). The porosity of beta-TCP materials, both macroscopic and microscopic, is designed to maximize blood clot stability during early healing. The material has no organic components, and therefore, there is no chance of antigenicity or allergic reactions (32).

The novelty of the presented technique is in the attempt to prevent the collapse of buccal, lingual and crestal bone tissues just by applying bone fillers, stabilizing the blood clot and avoiding soft tissue ingrowth. Afterwards, together with systemic antibiotics, undisturbed wound healing and reossification are expected.

In this context, three approaches were combined, which provided a therapeutic benefit by reducing the periodontal pocket in the mandible, eliminating pathogenic microorganisms, extracting the tooth, retracting the deep periodontal pocket on the adjacent tooth, and using GTR and systemic antibiotics to offer a prophylactic benefit to the patient in the future. It will be helpful to aim prefer- ably for primary care clinics to make the current trends in patient care available to observation, and at the same time to teach our students the skills they particularly need for outpatient care (33).

This one-stage treatment approach reduced the costs of untreated aggressive periodontitis and prevented the potential loss of periodontitis-affected adjacent teeth caused by the growth of the third mandibular molar.

\section{Conclusion}

Adequate periodontal assessment and treatment, appropriate instructions and compliance with maintenance protocols appear to be the most important factors in limiting or avoiding the potential negative effects on the periodontium caused by a horizontally impacted mandibular molar.

We are using the results of this study in an ongoing project that is focused on the progressive and innovative methodology for conservative and surgical periodontal treatments, surgical treatment of impacted mandibular third molars and GTR of adjacent second molars in a one-stage treatment approach. The main idea of this new approach is to use and combine the advantages of periodontal treatment with systemic antibiotics after identifying the bacteria, and surgical treatments that also require systemic antibiotics.

The complex periodontal and oral surgical procedure can be performed in one day with systemic antibiotics for both procedures together, as in the OSFMD approach. This approach eliminates the risks of destroying the bone during the surgical extraction of $3 \mathrm{M}$, promotes bone formation and maturation after GTR, and prevents aggressive periodontitis in the future.

The purpose of this unique study is to report the clinical results 10 years after the primary treatment. The results showed the importance of obtaining accurate diagnoses for the patient, summarizing them and developing a definite treatment plan. The described approach had good results over 10 years in maintaining the patient's periodontal health over time while being optimally cost effective.

The one-stage treatment approach appears to protect periodontal health in general, as well as protects the second adjacent mandibular molar from periodontal breakdown following the surgical extraction of $3 \mathrm{M}$ with GTR.

\section{References}

1. Lang NP, Bartold PM. Periodontal health. J Clin Periodontol 2018; 45 (Suppl 20): S9-S16. https:// doi.org/10.1111/jcpe.12936

2. Papapanou PN, Sanz M et al. Periodontitis: Consensus report of Workgroup 2 of the 2017 World Workshop on the Classification of Periodontal and Peri-Implant Diseases and Conditions. J Clin Periodontol 2018; 45 (Suppl 20): S162-S170. https://doi.org/10.1111/jcpe.12946

3. Lindhe $\mathbf{J}$, Lang NP, Berglundh $\mathbf{T}$ et al. Clinical periodontology and implant dentistry. Sixth edition. Ames, Iowa: John Wiley and Sons, 2015.

4. Blieden TM. Tooth-related issues. Ann Periodontol 1999; 4: 91-97.

5. Alessandri Bonetti G, Incerti Parenti S, Checchi L. Orthodontic extraction of mandibular third molar to avoid nerve injury and promote periodontal healing. J Clin Periodontol 2008; 35 (8): 719-723 [cit 202002-27]. DOI: 10.1111/j.1600-051X.2008.01286.x. ISSN 03036979. 
796-800

6. Fine DH, Patil AG, Loos BG. Classification and diagnosis of aggressive periodontitis. J Clin Periodontol 2018; 45 (Suppl 20): S95-S111. https:// doi. org/10.1111/jcpe.12942.

7. Ercoli C, Caton JG. Dental prostheses and tooth-related factors. J Clin Periodontol 2018; 45 (Suppl 20): S207-S218. https://doi.org/10.1111/ jcpe. 12950.

8. O’Leary TJ, Drake RB, Naylor JB. The Plaque Control Record. J Periodontol 1972; 43 (1): 38-38 [cit 2020-03-15]. DOI: 10.1902/ jop.1972.43.1.38. ISSN 0022-3492.

9. Ainamo J, Bay I. Problems and proposals for recording gingivitis and plaque. Internat Dent J 1975; 25 (4): 229-235.

10. Armitage GC. Periodontal diagnoses and classification of periodontal diseases. Periodontology 2004; 34: 9-21.

11. Xajigeorgiou C, Sakellari D, Slini T, Baka A, Konstantinidis A. Clinical and microbiological effects of different antimicrobials on generalized aggressive periodontitis. J Clin Periodontol 2006; 33: 254-264.

12. Quirynen M, Bollen CML, Vandekerckhove BN, Dekeyser C, Papaioannou W, Eyssen H. Full- versus partial-mouth disinfection in the treatment of periodontal infection. Short-term clinical and microbiological observations. J Dent Res 2005; 74: 1459-1467.

13. Teughels W, Dekeyser C, Van Essche M, Quirynen M. One-stage, full-mouth disinfection: fiction or reality? Periodontology 2009; 50: 39-51.

14. Quirynen M, Teughels W, van Steenberghe D. Impact of antiseptics on one-stage full-mouth disinfection. J Clin Periodontol 2006; 33: 49-52.

15. Muller HP, Heinecke A, Borneff M, Kiencke C, Knopf A, Pohl S. Eradication of Actinobacillus actinomycetemcomitans from the oral cavity in adult periodontitis. J Periodont Res 1998; 33: 49-58.

16. Haffajee AD, Socransky S, Gunsolley J. Systemic anti-infective periodontal therapy. A systematic review. Ann Periodontol 2003; 8: 115-181.

17. Herrera D, Alonso B, Leo`n R, Rolda `n S, Sanz M. Antimicrobial therapy in periodontitis: the use of systemic antimicrobials against the subgingival biofilm. J Clin Periodontol 2008; 35 (Suppl 8): 45-66.

18. Walker CB, Gordon JM, Magnusson I, Clark WB. A role for antibiotics in the treatment of refractory Periodontitis. J Periodontol 1993; 64: 772 .

19. Mestnik MJ, Feres M, Figueredo LC, Duarte PM, Lira EAG, Faveri M. Short-term benefits of the adjunctive use of metronidazole plus amoxicillin in the microbial profile and in the clinical parameters of subjects with generalized aggressive periodontitis. J Clin Periodontol 2008; 37: $353-365$.

20. Aimetti M, Romano F, Guzzi N, Carnevale G. Full-mouth disinfection and sytemicantimicrobial therapy in generalized aggressive periodontitis: a randomized, placebocontrolledtrial. J Clin Periodontol 2012; 39: 284-294. DOI: 10.1111/j.1600-051X.2011.01795.
21. Koniarova A, Sedlata Juraskova E, Spidlen M, Statelova D. The influence of orthodontic non-extraction treatment on the change in the inclination and position of incisors in the Europoid race. Bratisl Med J 2017; 118 (11): 662-668. DOI: 10.4149/BLL_2017_126.

22. Montero J, Mazzaglia G. Effect of removing an impacted mandibular third molar on the periodontal status of the mandibular second molar. J Oral Maxillofac Surg 2011; 69: 2691-2697.

23. Kan KW, Liu JK, Lo EC, Corbet EF, Leung WK. Residual periodontal defects distal to the mandibular second molar 6-36 months after impacted third molar extraction. J Clin Periodontol 2002; 29: 1004-1111.

24. Sammartino G, Tia M, Bucci T, Wang HL. Prevention of mandibular third molar extraction-associated periodontal defects: A comparative study. J Periodontol 2009; 80: 389-396.

25. Ziegler RS. Preventive dentistry - New concepts. Preventing periodontal pockets. Va Dent J 1975; 52: 11-13.

26. Kugelberg CF. Periodontal healing two and four years after impacted lower third molar surgery. A comparative retrospective study. Int J Oral Maxillofac Surg 1990; 19: 341-345.

27. Alqahtani, NA, Khaleelamed, A, Desai, F. Evaluation of two flap designs on the mandibular second molar after third molar extractions. J Oral Maxillofac Pathol 2017; 21 (2). DOI: 10.4103/jomfp.JOMFP_75_17.

28. BlancoGD, Lora Marzola C. The Different Types of Flaps in the Surgical Relations of the Third Impacted Molars-Literature Review. Dentistry 2017; 7 (4). DOI: 10.4172/2161-1122.1000425.

29. Lopez JL. The Importance of Flap Design in Third Molar Surgery: A systematic Review. Biomed J Sci Techn Res 2018; 11 (5). DOI: 10.26717/ BJSTR.2018.11.002164.

30. Pecora G, Celetti R, Davarpanah M, Covani U, Etienne D. The effect of guided tissue regeneration on healing after impacted third molar surgery: 1-year results. Internat J Periodont Restor Dentistry 19993; 13: 397-407.

31. Karapataki S, Hugoson A, Kugelberg CF. Healing following GTR treatment of bone defects distal to the 2nd molars after surgical removal of impacted 3rd molars. J Clin Periodontol 2000; 27: 325-332.

32. Tadic D, Epple M. A thorough physicochemical characterisation of 14 calcium phosphate-based bone substitution materials in comparison to natural bone. Biomaterials 2004; 25 (6): 987-994.

33. Ostatnikova D, Hopkins MA, Riles T, Robinson E, Hnilicova S. Universities and their educational mission in the 21st century: Medical education innovations at Comenius University Faculty of Medicine. Bratisl Med J 2019; 120 (9): 617-620. DOI: 10.4149/BLL_2019_101.

Received April 13, 2020. Accepted June 15, 2020. 